\title{
The chronicity and severity of abuse among older persons by country: a European study
}

\author{
Joaquim J.F. Soares, Silvia Fraga, Eija Viitasara, Mindaugas Stankunas, Örjan Sundin, \\ Maria Gabriella Melchiorre, Gloria Macassa and Henrique Barros
}

\author{
Joaquim J.F. Soares is \\ a Professor, Dr Eija Viitasara is \\ a PhD, Senior Lecturer and \\ Dr Gloria Macassa is an \\ Associate Professor, all are \\ based \\ at Public Health Sciences, \\ Mid Sweden University, \\ Sundsvall, Sweden. \\ Dr Silvia Fraga is a $\mathrm{PhD}$, based \\ at Department of Clinical \\ Epidemiology, University of \\ Porto Medical School, Porto, \\ Portugal. \\ Mindaugas Stankunas is \\ a Professor, based at \\ Department of Health \\ Management, Lithuanian \\ University of Health Sciences, \\ Kaunas, Lithuania. \\ Örjan Sundin, is a Professor, \\ based at Social Sciences - \\ Psychology Östersund, \\ Mid Sweden University, \\ Östersund, Sweden. \\ Maria Gabriella Melchiorre is \\ a Senior Researcher - \\ Gerontologist, based at Italian \\ National Institute of Health and \\ Science on Aging, Ancona, \\ Italy. \\ Henrique Barros is a Full \\ Professor, based at Medical \\ School - Hygiene and \\ Epidemiology, University of \\ Porto, Porto, Portugal.
}

\begin{abstract}
Purpose - The purpose of this paper is to investigate chronicity (frequency) in different abuse types (e.g. psychological) and overall abuse (all abuse types) by severity (minor, severe, total) in seven European cities, and scrutinize factors associated with high chronicity levels (frequency on the median and higher) in psychological and overall abuse by severity.

Design/methodology/approach - The study design was cross-sectional. The sample consisted of 4,467 randomly selected women/men (2,559 women) aged 60-84 years from seven European cities, and data were analysed with bivariate and multivariate methods.

Findings - Chronicity varied across country and by abuse type. For instance, Germany had the highest chronicity means in physical and sexual abuse; Greece in physical, injury, sexual and overall abuse; Lithuania in physical, injury, financial and overall abuse; Portugal in physical abuse; Spain in physical, sexual and financial abuse; and Sweden in psychological, injury, financial and overall abuse. In general, Italy had the lowest chronicity means. The main perpetrators were people close to the respondents and women (in some cases).

Research limitations/implications - The independent relationship (regressions) between chronicity/ severity of abuse, country and other variables (e.g. depression) was examined only for psychological and overall abuse. More research into this issue with other types of abuse (e.g. sexual) is warranted.

Originality/value - The paper reports data from the ABUEL survey, which gathered population-based data on elderly abuse.

Keywords Mental health, Abuse, Chronicity, Country, Older persons

Paper type Research paper

\section{Introduction}

Older age is often associated with strains such as increased morbidity, loneliness and reduced financial capacity (e.g. Boyd et al., 2005; Buber et al., 2010; Cornwell and Waite, 2009; Crimmins and Beltrán-Sánchez, 2011; Dykstra et al., 2005; Lelkes and Gasior, 2012; Watson et al. , 2010)[1], and abuse may be an additional stress factor increasing the total burden of older persons (Sethi et al., 2011). A review (Cooper et al., 2008) of 49 studies with different samples and recent studies with general population/community samples (e.g. Acierno et al., 2010; Lindert et al., 2013; Laumann et al., 2008; Lowenstein et al., 2009; Macassa et al., 2013; O'Keeffe et al., 2007) concerning abuse against older persons revealed prevalence rates between 0.6 and 55 per cent, with verbal and any abuse as high as 52 and 55 per cent, respectively. Elder abuse co-exists with for instance injury, depression, isolation and premature mortality (e.g. Abath et al., 2010; Acierno et al., 2010; Dong and Simon, 2008; Dong et al., 2009, 2013; Lachs et al., 1998; Laumann et al., 2008; Lowenstein et al., 2009; Melchiorre et al., 2013). Elder abuse is indeed associated with adverse outcomes (e.g. depression) and
\end{abstract}


once considered as a "social welfare issue and a problem of ageing" (WHO, 2002), is nowadays a major public health issue.

However, there is limited information about the patterns of abuse across countries or cultures. As far as we know, only four studies have addressed the prevalence of elder abuse with national samples (Lindert et al., 2013; Luoma et al., 2011; Macassa et al., 2013; O'Keeffe et al., 2007). The ABUEL study (Lindert et al., 2013; Macassa et al., 2013), based on data of 4,467 women/ men aged 60-84 years from seven European countries (Germany, Greece, Italy, Lithuania, Portugal, Spain, Sweden), reported abuse prevalence rates across countries between 0.7 and 19.4 per cent. The highest rates of psychological and physical abuse were found in Sweden (29.7 and 4 per cent), of sexual abuse in Greece (1.5 per cent), of financial abuse in Portugal (7.8 per cent) and of injury in Lithuania (1.5 per cent), and in general there were no differences between women/men. The Abuse and Violence Against Older Women (AVOW) study (Luoma et al., 2011), based in a sample of older women aged 60 years and over from five European countries (Austria, Belgium, Finland, Lithuania, Portugal), showed prevalence rates ranging between 0.5 and 32.9 per cent depending on the abuse type. Belgium and Portugal had the highest rates of overall abuse (32 and 39.4 per cent), but also in most abuse types (e.g. psychological, 27.5 and 32.9 per cent). The UK study (O'Keeffe et al., 2007), with data only from UK regions (England, Northern Ireland, Scotland, Wales), of elder abuse among women/men aged 66 years and older reported abuse rates ranging from 0.2 to 1.1 per cent. The lowest rate was sexual abuse ( 0.3 per cent) and the highest neglect ( 1.1 per cent). Wales had the highest rates (3.1 per cent) and North Ireland the lowest ( 2 per cent), and women reported more abuse than men (3.8 vs 1.1 per cent) The observed discrepancies in for instance prevalence rates between these studies are large, particularly concerning the UK study. This may be explained by some methodological options. For example, in the UK study an act of psychological abuse was considered only if it occurred ten or more times. Thus, it is possible that prevalence of abuse in the UK was underestimated, at least regarding psychological abuse. In the case of the AVOW study the observed differences may be due to for instance the sampling approach since it used only women and included respondents above 84 years.

In general there were greater prevalence rates of abuse in northern than in southern countries in the ABUEL study (Lindert et al., 2013; Macassa et al., 2013) and no differences between women/men, while the opposite was to some extent found with regard to prevalence in the AVOW study with only women (Luoma et al., 2011). Data on homicides among older persons committed by family members and studies on intimate partner violence (IPV) indicate higher homicide and violence rates in male dominated, southern and/or developing countries (e.g. Sethi et al., 2011; Straus and Mickey, 2012; WHO, 2002). Additionally, Archer (2006) in a cross-cultural study found that violence rates against women were higher in countries with low gender empowerment and gender-related development index. A recent study in six European countries (England, Germany, Greece, Hungary, Portugal, Sweden) on the prevalence/chronicity of IPV victimization/perpetration among women/men (Costa et al., 2013)[2], found for instance that the chronicity of most types of IPV was higher in southern (e.g. Greece) than in northern countries (e.g. Sweden), and in general there were no differences between women/men. Furthermore, although prevention and information interventions concerning elder abuse have been/are applied in for instance Europe, this may be more evident in Northern and highly developed countries (Sethi et al., 2011).

As indicated earlier there was an excess of abuse prevalence in northern compared to southern countries in our data (Lindert et al., 2013; Macassa et al., 2013) , but the situation may be different regarding the chronicity of abuse (frequency) by severity in view of other findings (e.g. IPV), prevention/information actions and human development indexes (HDIs) (UNDP, 2013). In line with this, a reverse pattern is conceivable concerning chronicity, i.e. higher percentages in southern than in northern countries. As to gender differences in chronicity, no firm conclusions can be made in view that there is only one study (IPV) available with general population samples (Costa et al., 2013).

Although two studies have been conducted with regard to chronicity (Costa et al., 2013; Luoma et al., 2011), the chronicity (frequency) and severity (minor, severe) of violence as operationalized by the Conflict Tactic Scales 2 (CTS2, Straus et al., 1996) has attracted little attention concerning 
older persons, despite that several authors have stressed the importance of such data (Bennett and Kingston, 1993; Luoma et al., 2011). Thus, there is a scarcity of comparative data on the patterns of elder abuse between different countries or cultures concerning different abuse types, particularly concerning the chronicity of different abuse types and overall abuse by severity among women/men (age 60-84 years) from the general population, and which factors influence chronicity of abuse. Little is known also on gender differences in chronicity/severity among this type of population. Such information could provide new insights into for instance the patterns of abuse, particularly chronicity/severity, in different countries/cultures and contribute to the development of effective advocacy and actions tailored after chronicity/severity patterns with the ultimate aims of protecting abused older persons and those at risk.

Accordingly, the main aims of this study were: to describe the chronicity (frequency) of different abuse types (e.g. psychological) and overall abuse by severity (minor, severe, total) in a sample of women/men (60-84 years) from the general population of seven European cities that disclosed their abuse experiences during the past 12 months; and to identify/quantify factors associated with chronicity by severity of psychological and overall abuse in a sample of women/men (60-84 years) from the general population of seven European cities that disclosed their abuse experiences during the past 12 months.

\section{Methods \\ Sample}

The sample in the ABUEL study were women/men living in seven European cities (Stuttgart, Germany; Athens, Greece; Ancona, Italy; Kaunas, Lithuania; Porto, Portugal; Granada, Spain; Stockholm, Sweden). Inclusion criteria were not suffering from cognitive (e.g. dementia) or sensory (e.g. blindness) impairments, living in own/rented housing or homes for elderly and able to read/write or express themselves in the native languages (see Lindert et al., 2012, 2013; Macassa et al., 2013; Melchiorre et al., 2013 for more details).

The sample size was calculated based on municipal census in each city and expected prevalence rates of abuse reported in previous surveys. Departing from a mean abuse prevalence of 13 per cent, with a precision of 2.6 per cent, derived from a recent review (Cooper et al., 2008), a sample size of 633 persons in each city was required. A maximum of 656 persons was permitted for each city considering the infinite population assumption. The sample size was adapted to each city according to their total population of women/men aged 60-84 years. The respondents were selected through random stratification by sex and age[3]. The overall and final sample comprised 4,467 older persons (2,559 women, 57.3 per cent), with a mean response rate across countries of 45.2 per cent. Detailed information on the study design, sampling and assessments methods; on the target population by country, sex and age; on the persons eligible, cooperation, completion and response rates by country; on the population fraction (PF) and population fraction ratio (PFR)[4] by country, sex and age in relation to the reference population; and on the analyses (regression) of refusal data by country, sex and age are shown in a separate ABUEL method paper (Lindert et al., 2012). Overall, contrasts of the respondents with the reference population in the community census database (age/sex) and refusal data (excluding Greece), revealed that in Portugal more women than men responded to the survey and in Italy more women than men declined to participate. The youngest groups in all cities more often declined to participate. There were no other differences (see also Lindert et al., 2012). Details on demographics/socio-economics are presented in detail elsewhere (Lindert et al., 2013; Macassa et al., 2013; Melchiorre et al., 2013).

\section{Measures}

Abuse was measured with 52 questions based on the CTS2 (Straus et al., 1996) and on the UK survey of elder abuse/neglect (O'Keeffe et al., 2007). Psychological abuse comprised 11 questions, of which six were severe acts (e.g. threatened with being hit or having something thrown at)[5] and five minor (e.g. shouted or yelled at); physical abuse 17 questions, of which ten were severe acts (e.g. burned or scalded) and seven minor (e.g. being grabbed); injury seven questions, of which four were severe acts (e.g. passed out from being hit on the head) and three 
minor (e.g. had a sprain, bruise or small cut from being hit); sexual abuse eight questions, of which five were severe acts (e.g. had sexual intercourse with you against your will) and three minor (e.g. tried to touch you in a sexual way against your will); financial abuse nine questions, of which five were severe acts (e.g. made you give him/them your money, possessions or property against your will) and four minor (e.g. tried to make you give money, possessions or property). The frequency of abuse acts was expressed in terms of: happened once (1), twice (2), three to five (midpoint 4), six to ten (midpoint 8), 11-20 (midpoint 15) or $>20$ (25) times during the past year (chronicity), had not happened in the past year, but before that or never. When respondents answered that they had not been abused during the past year, they were considered as no abuse cases (no, 0). If respondents answered that they had been abused during the past year, they were considered as abuse cases (yes, 1). The present study focused on chronicity (frequency of acts) by severity (minor, severe, total)[6] of each abuse type (e.g. psychological) and overall abuse (all types) during the past year.

Departing from the frequency of abuse acts $(1,2,4,8,15,25)$ of the total abused population, medians of chronicity by severity (minor, severe, total) of each abuse type and overall abuse were calculated. Thereafter, chronicity was dichotomized in low (frequency of abuse acts under median) and high (frequency of abuse acts on the median and higher). Additionally, the frequency of minor and severe acts of each abuse type and overall abuse was pooled together into a total chronicity, but also dichotomized (low total chronicity, minor/severe acts together under median; high total chronicity, minor/severe acts together on the median and higher). The Cronbach $\alpha$ 's across the total population were psychological abuse 0.85 , physical abuse 0.80 , injury 0.72, sexual abuse 0.76 and financial abuse 0.71 .

Depression and anxiety were measured with the Hospital Anxiety and Depression Scale (Zigmond and Snaith, 1983). It comprises 14 questions (graded 0-3), of which seven involve depression (e.g. lost interest in appearance) and seven anxiety (e.g. sudden feelings of panic). The total score for depression and anxiety is 21 (each). High scores correspond to high depression/anxiety levels. Scores 0-7 correspond to no cases, 8-10 to possible cases and 11-21 to probable cases. In this study, the data were dichotomized into no cases (scores 0-7) and cases (scores 8-21) of depression/anxiety. Cronbach's $\alpha$ for anxiety across countries was 0.81 and for depression 0.80 .

Somatic symptoms were measured with the short version of the Giessen Complaint List (Gunzelmann et al., 1996). It comprises 24 questions (graded 0-4, not affected-very much affected). The symptoms are divided in four domains (six questions in each): Exhaustion (e.g. tiredness), gastrointestinal (e.g. nausea), musculoskeletal (e.g. pains in joints or limbs) and heart distress (e.g. heavy, rapid or irregular heart-throbbing), and a total score can be extracted (96). The higher the scores, the more one is affected. Based on median across countries, total scores were dichotomized into low (below median) and high (median and above) symptom levels. Cronbach's $\alpha$ for somatic symptoms across countries was 0.92 .

Social support was measured with the Multidimensional Scale of Perceived Social Support (Zimet et al., 1990). It comprises 12 questions (graded 1-7) arranged into three categories (support from family, significant others and friends). The total score amounts to 84. High scores correspond to high social support. Based on median across countries, social support was dichotomized in low (below median) and high (median and above) levels of social support. Cronbach's $\alpha$ for social support across countries was 0.92 .

Leisure activities were assessed with 13 questions about the frequency to which respondents had been involved in various activities (e.g. meeting friends) during the past year. The frequency ranged from never to daily. The questions were based on the Stockholm County Council health survey (Centrum för folkhälsa. Stockholms läns landsting, 2007).

Health care use was assessed as the number of contacts with different health care staff (e.g. physician) and health care services (e.g. primary care) the past year. Further, we assessed the number of diseases (e.g. cardio-vascular), which the respondents were presently suffering from. The questions were based on the Stockholm County Council health survey (Centrum för folkhälsa. Stockholms läns landsting, 2007). 
Alcohol use was assessed with questions derived from the Alcohol Use Disorders Identification Test (Babor et al., 2001). First the respondents were asked if they presently used alcohol (do you drink alcohol?, yes/no). If they answered yes, three items derived from audit were asked: how often do you have a drink containing alcohol? (once a month or less, two to four times a month, two to three times a week, four or more times a week); how many drinks containing alcohol do you have on a typical day when you are drinking? ( 1 or 2,3 or 4,5 or $6,7,8$, or 9,10 or more); how often do you have six or more drinks on one occasion? (never, less than monthly, monthly, weekly, daily or almost daily). Finally, respondents were asked about their previous use of alcohol (if you do not drink alcohol now, have you ever drunk alcohol? yes/no). This study focused on whether the respondents used alcohol or not[7].

Demographics/socio-economics were assessed and this study focused on the following variables: country, age, sex, marital status, education, profession, financial support, financial strain, household size and if still in work. Financial strain (preoccupation with how to make ends meet) was assessed with one item (no/sometimes/often/always format). A respondent was considered to experience "financial strain" if she/he selected any response other than no. Four items (e.g. birth place) assessed whether the respondents were migrants or indigenous inhabitants. The demographics/socio-economics were tailored for each country, but similar in content.

\section{Design/procedure}

The design was cross-sectional. Recruitment and data collection in the seven European cities were performed during January-July 2009. The data were collected through face-to-face interviews (on average one hour) of the respondents usually in their homes and making sure that they were alone. In general, the respondents were first contacted by telephone/letter and then an appointment was made. The interviews were conducted by previously trained female interviewers following an interview manual (www.abuel.org/). In several cities, the data were collected through a combination of face-to-face interviews and self-response[8]. All measures (if not available) were translated into the native languages, back translated and culturally adapted, and the same procedure was used for other materials (e.g. information letters). The respondents were informed (in writing/verbally) about the research and informed consent was requested. Confidentiality, anonymity and the participant's rights were emphasized. The respondents could stop the data collection at any point in time. Ethical permission was received in each country (for further details see Lindert et al., 2012, 2013; Macassa et al., 2013; Melchiorre et al., 2013).

\section{Statistical analyses}

Bivariate/multivariate analyses with regard to the prevalence of abuse (e.g. psychological) and social support considering other variables (e.g. demographics), descriptions of perpetrators and background variables of the respondents are shown elsewhere (Lindert et al., 2013; Macassa et al., 2013; Melchiorre et al., 2013).

All analyses in the present study were conducted on respondents exposed to abuse during the past 12 months. The data on chronicity (frequency of abuse acts) by severity (minor, severe, total) of each abuse type and overall abuse by country were shown as means, standard deviations and 95 per cent confidence intervals (95 per cent $\mathrm{Cl}$ ). Differences between countries in chronicity by severity of each abuse type and overall abuse were examined with $\chi^{2}$ testes, following dichotomisation (see measures). The significant level for bivariate and multivariate analyses was set at $p<0.05$.

Furthermore, six multiple block-wise logistic regression analyses, three each for chronicity (frequency) by severity (minor, severe, total) of psychological and overall abuse were conducted. In block-wise logistic regression, variables are inserted into the regression equation block by block and the contribution of every block in explaining the dependent variable is expressed as Nagelkerke $R^{2}$ changes. Nagelkerke $R^{2}$ (Peng et al., 2002) is an approximation to descriptive goodness-of-fit statistics to examine if the proposed logistic model fits (the strength of association between variables is quantified). 
The dependent variables were chronicity by severity of psychological abuse and overall abuse dichotomized into low (under median) and high (median and higher) levels. The independent variables were selected based on statistical inference, i.e. factors (e.g. socio-economics) that differentiated abused/non-abused respondents in previous analyses (Lindert et al., 2013; Macassa et al., 2013; Melchiorre et al., 2013). The independent variables comprised country, age, sex, marital status, education, profession, financial support, still in work, alcohol use, household size, somatic symptoms (low/high), possible/probable cases of depression and anxiety, diseases number, health care use, leisure activities (frequency) and social support (low/ high). Additionally, we added type of perpetrator (e.g. children/grandchildren) and sex of the perpetrator. Due to the limited number of cases in physical, injury, sexual and financial abuse regressions were not conducted on these abuse types separately. However, these abuse types were included in the regression of overall abuse. Associations between variables were expressed as odds ratio and 95 per cent $\mathrm{Cl}$ and Nagelkerke $R^{2}$. Analyses were carried out using the SPSS statistical package 20 (IBM Corporation, 1989/2011)

\section{Results}

\section{Bivariate analyses of abuse chronicity}

Psychological. As shown in Table I, the means of chronicity (frequency) by severity (minor, severe, total) were elevated in most countries, particularly in Greece, Lithuania, Portugal, Spain and Sweden. However, of all countries, Lithuania and Sweden had the greatest percentages of high chronicity levels (median and higher) independent of severity. Thus, respondents in most countries were exposed to many acts of psychological abuse by severity form, particularly those in Lithuania and Sweden in relationship to high chronicity levels.

Physical and injury. Of all countries, Greece, Lithuania and Portugal tended to have the highest means in chronicity (all severity forms), but they were particularly distinguishable with regard to the percentages in high chronicity levels. Italians had not been exposed to high levels of chronicity in physical abuse (severe, total) and did not sustained injuries at all, and Germans were not exposed to severe injury and show no high chronicity levels (total). Thus, respondents in Greece, Lithuania and Portugal sustained many acts of physical abuse and injury.

Sexual. Germany, Greece, Portugal and Spain had high means in chronicity (most severity forms), and in particular Germany. However, of all countries, Greece and Portugal had the greatest percentages with regard to high levels of chronicity by severity of sexual abuse. There were no cases of high levels of chronicity among Swedes (severe), and Italians (severe, total) and Lithuanians had not been exposed to severe sexual abuse. Notwithstanding, the differences between countries were not significant.

Financial. With the exception of Sweden, means of financial abuse chronicity (all severity forms) were relatively low. However, Greece, Lithuania and Portugal tended to have the greatest percentages of high chronicity levels by severity indicating that respondents in these countries had been exposed to many episodes of financial abuse. Italians had the lowest levels of high chronicity.

Overall. Means of chronicity (all severity forms) were general high in all countries, particularly in Greece, Lithuania and Sweden. However, Germany, Lithuania and Sweden had the greatest percentages of high chronicity levels in all severity forms. Thus, respondents in these countries were more prone to be exposed to many abusive acts across abuse types and severity.

\section{Multivariate analyses of high abuse chronicity}

Psychological, minor. As shown in Table II, in the demographic/socio-economic block, respondents from Germany, Greece, Italy, Portugal and Spain in relation to the reference country (Sweden) and single in relation to married/cohabitants were less likely to have been exposed to high levels of minor chronicity, and females more likely. The block accounted for 10.4 per cent of the total variance in minor chronicity. The block life-style, using alcohol, was not independently associated with minor chronicity and it accounted for 0.1 per cent of the total variance. In the block health indicators, suffering from possible/probable cases of anxiety and depression were 


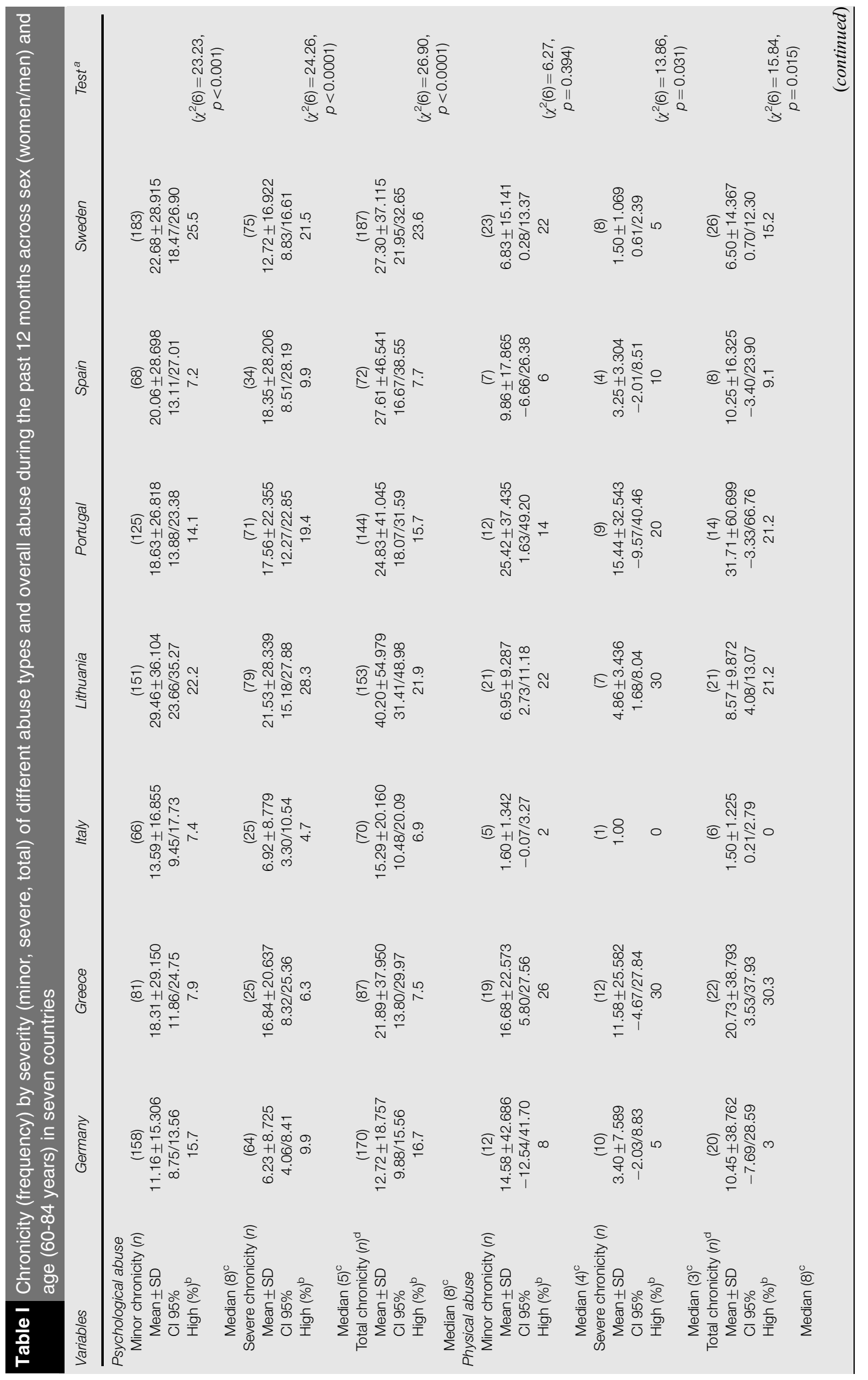




\begin{tabular}{|c|c|c|c|c|c|c|c|c|}
\hline 离 & $\underset{d}{\stackrel{f}{f}}$ & $\begin{array}{l}\mathcal{m}^{-} \\
\text {II } \\
\text { IJ }\end{array}$ & $\begin{array}{l}10 \\
6 \\
11 \\
10 \\
0 \\
0 \\
0\end{array}$ & $\begin{array}{l}8 \\
0 \\
11 \\
0\end{array}$ & $\begin{array}{l}\hat{0} \\
0 \\
1 \\
0\end{array}$ & & & 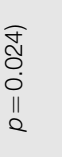 \\
\hline $\begin{array}{l}\text { क्षे } \\
\text { के } \\
\text { के }\end{array}$ & 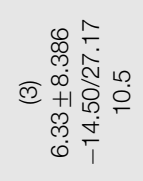 & 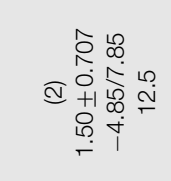 & 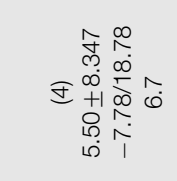 & 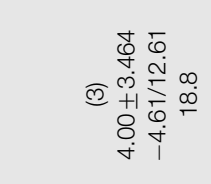 & 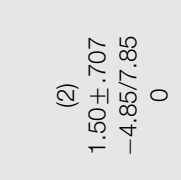 & 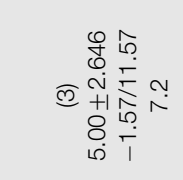 & 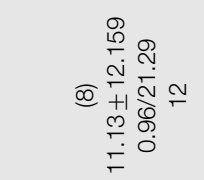 & \\
\hline $\begin{array}{l}\text { कू } \\
\text { का }\end{array}$ & 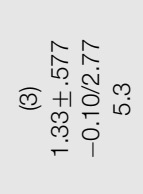 & 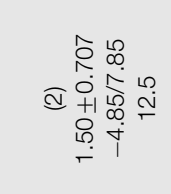 & 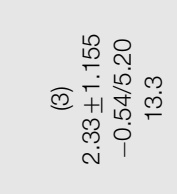 & 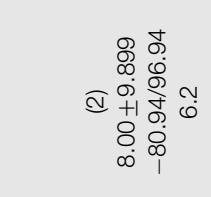 & 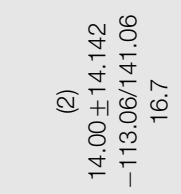 & 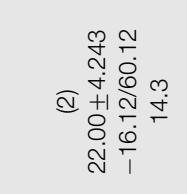 & 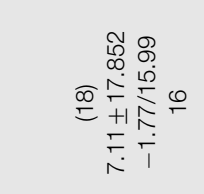 & \\
\hline $\begin{array}{l}\text { बू } \\
\text { है } \\
0\end{array}$ & 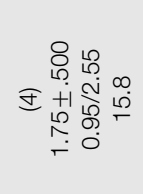 & 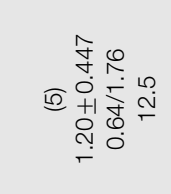 & 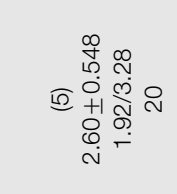 & 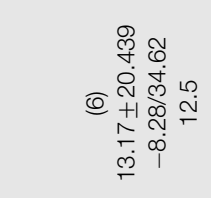 & 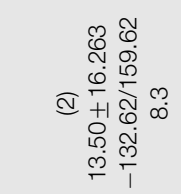 & 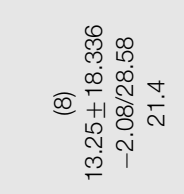 & 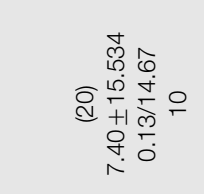 & \\
\hline 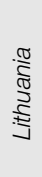 & 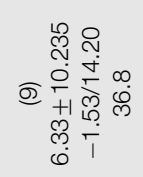 & 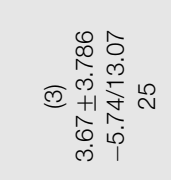 & 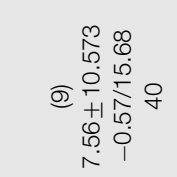 & 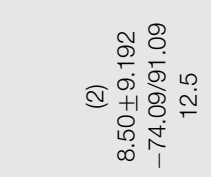 & & 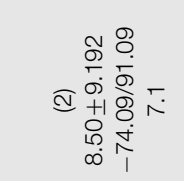 & 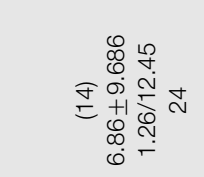 & \\
\hline હે & & & & 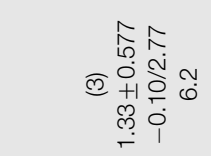 & E $\underset{+}{\stackrel{8}{r}} 0$ & 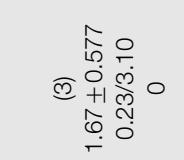 & 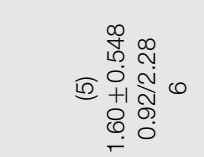 & \\
\hline 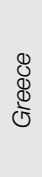 & 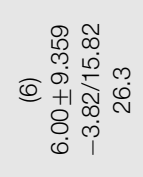 & 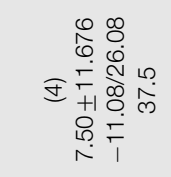 & 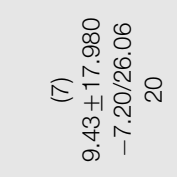 & 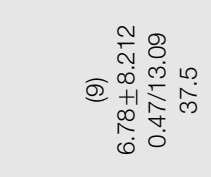 & 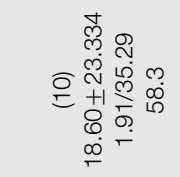 & 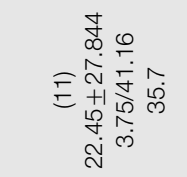 & 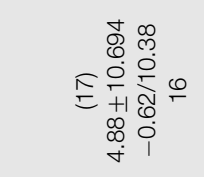 & \\
\hline $\begin{array}{l}\text { હે } \\
\text { है } \\
\text { ํ }\end{array}$ & 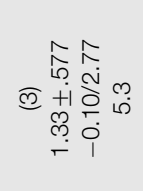 & & 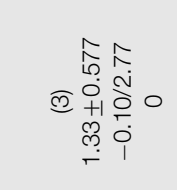 & 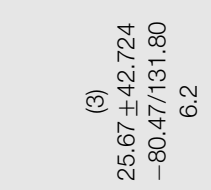 & 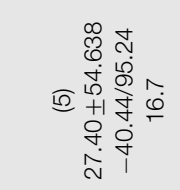 & 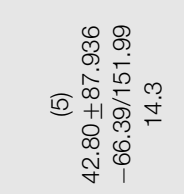 & 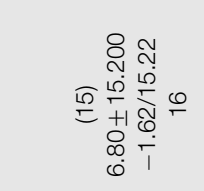 & \\
\hline$\frac{0}{\frac{0}{2}} \frac{\pi}{\frac{\pi}{m}}$ & 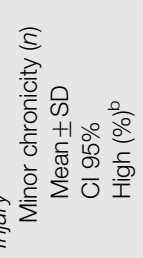 & 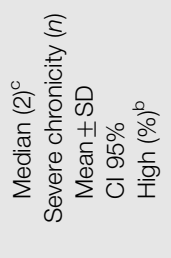 & 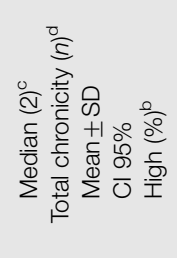 & 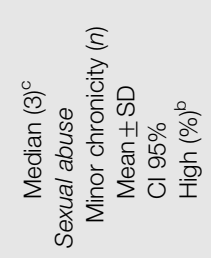 & 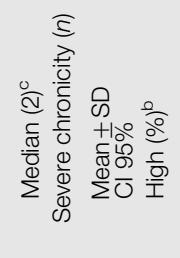 & 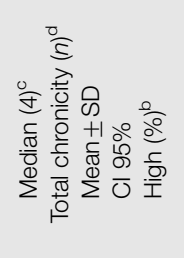 & 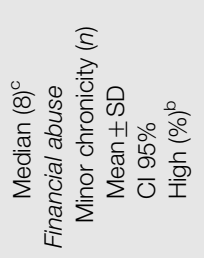 & 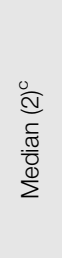 \\
\hline
\end{tabular}




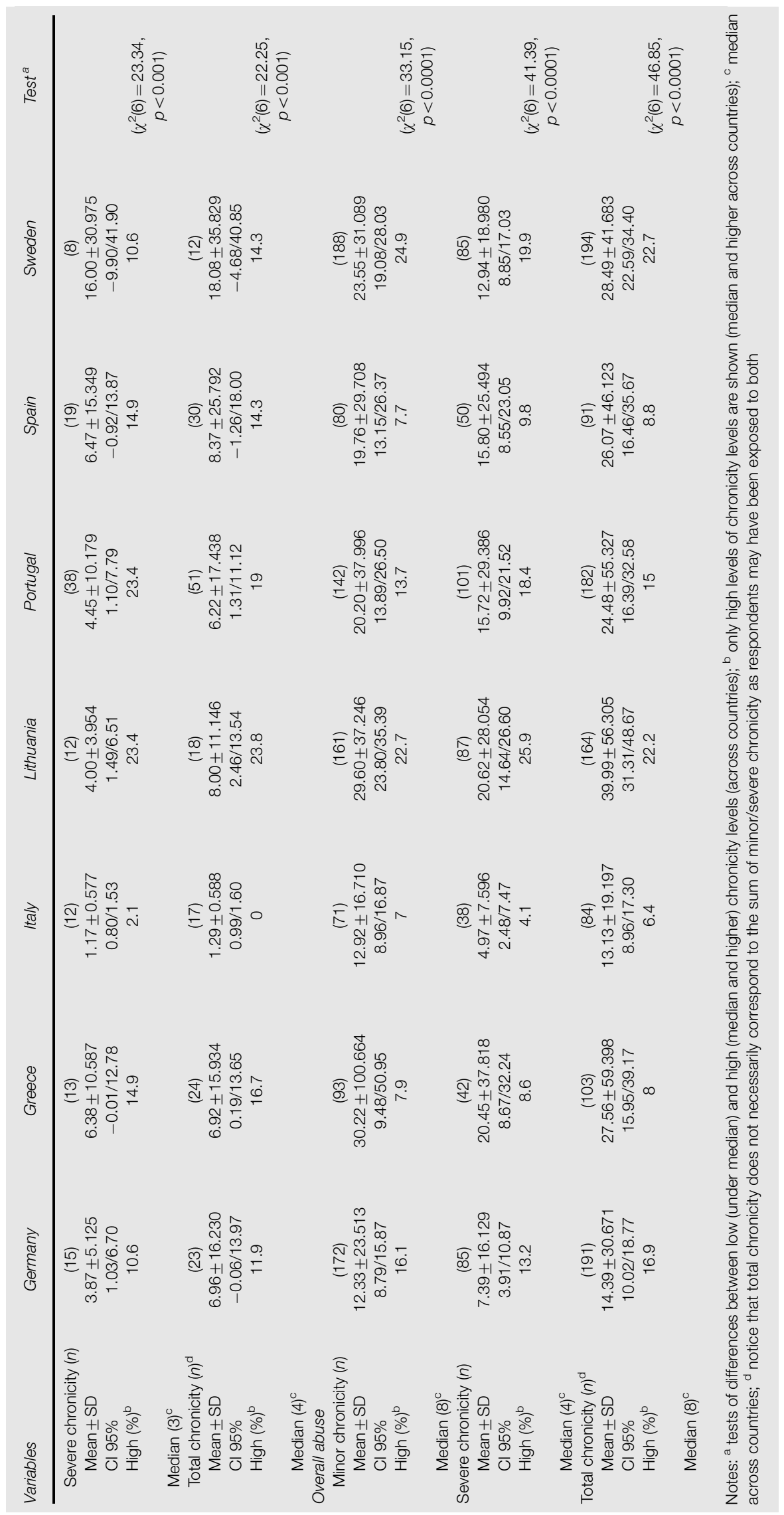


Table II Multiple block-wise logistic regression analyses of the association between demographics/socio-economics, life-style, health indicators, perpetrator factors, leisure activities, social support and high chronicity levels by severity of psychological abuse (odds ratio, $\mathrm{Cl} 95$ per cent, $\left.R^{2}\right)^{1}$

\begin{tabular}{|c|c|c|c|c|c|c|}
\hline \multirow[b]{2}{*}{ Independent variables } & \multicolumn{5}{|c|}{ Psychological abuse } & \\
\hline & OR & Cl 95\% & $O R$ & $\mathrm{Cl} 95 \%$ & $O R$ & $\mathrm{Cl} 95 \%$ \\
\hline \multicolumn{7}{|c|}{ Block I (demographics-socio-economics) } \\
\hline \multicolumn{7}{|c|}{ Country ${ }^{a}$} \\
\hline Germany & $0.391^{\star \star}$ & $0.218-0.703$ & $0.268^{*}$ & $0.093-0.773$ & $0.469^{\star \star}$ & $0.266-0.827$ \\
\hline Greece & $0.266^{\star \star \star}$ & $0.120-0.587$ & $0.210^{*}$ & $0.049-0.894$ & $0.264^{\star \star \star}$ & $0.122-0.572$ \\
\hline Italy & $0.452^{\star}$ & $0.222-0.922$ & 0.353 & $0.102-1.222$ & $0.455^{\star}$ & $0.227-0.909$ \\
\hline Lithuania & 0.738 & $0.403-1.351$ & 1.082 & $0.412-2.842$ & 0.965 & $0.530-1.760$ \\
\hline Portugal & $0.346^{\star \star \star}$ & $0.187-0.642$ & 0.441 & $0.178-1.092$ & $0.397^{\star \star}$ & $0.221-0.711$ \\
\hline Spain & $0.328^{\star \star}$ & $0.148-0.729$ & 0.464 & $0.143-1.511$ & $0.430^{\star}$ & $0.201-0.924$ \\
\hline Sweden ${ }^{\mathrm{b}}$ & 1 & & & & & \\
\hline \multicolumn{7}{|l|}{$\mathrm{Age}^{\mathrm{a}}$} \\
\hline 65-69 & 1.108 & $0.679-1.808$ & 1.139 & $0.504-2.572$ & 1.011 & $0.625-1.635$ \\
\hline $70-74$ & 0.802 & $0.478-1.347$ & 0.666 & $0.296-1.501$ & 0.823 & $0.498-1.363$ \\
\hline $75-79$ & 0.654 & $0.354-1.208$ & 0.576 & $0.229-1.444$ & 0.800 & $0.437-1.465$ \\
\hline $80-84$ & 0.569 & $0.282-1.148$ & 1.319 & $0.428-4.062$ & 0.639 & $0.330-1.238$ \\
\hline $60-64^{b}$ & 1 & & & & & \\
\hline \multicolumn{7}{|l|}{$\operatorname{Sex}^{\mathrm{a}}$} \\
\hline Female & $1.652^{*}$ & $1.099-2.484$ & $2.024^{*}$ & $1.065-3.848$ & $1.511^{*}$ & $1.019-2.241$ \\
\hline Male & 1 & & & & & \\
\hline \multicolumn{7}{|l|}{ Marital status ${ }^{a}$} \\
\hline Single & $0.351^{\star \star}$ & $0.158-0.782$ & 0.412 & $0.112-1.523$ & 0.572 & $0.265-1.234$ \\
\hline Divorced-separated & 1.136 & $0.620-2.080$ & 1.432 & $0.606-3.387$ & 1.076 & $0.600-1.931$ \\
\hline Widow-er & 0.604 & $0.360-1.015$ & 0.551 & $0.239-1.269$ & 0.689 & $0.416-1.142$ \\
\hline Married-cohabitant ${ }^{\mathrm{b}}$ & 1 & & & & & \\
\hline Household size $e^{c, d}$ & 1.095 & $0.890-1.348$ & 1.124 & $0.832-1.520$ & 1.142 & $0.927-1.405$ \\
\hline \multicolumn{7}{|l|}{ Education $^{\mathrm{a}}$} \\
\hline Low $^{\mathrm{e}}$ & 1.253 & $0.707-2.221$ & 1.190 & $0.491-2.880$ & 1.328 & $0.760-2.320$ \\
\hline Middle $e^{f}$ & 1.133 & $0.705-1.820$ & 0.885 & $0.436-1.794$ & 1.069 & $0.675-1.691$ \\
\hline High $^{\text {b, g }}$ & 1 & & & & & \\
\hline \multicolumn{7}{|l|}{ Profession $^{a}$} \\
\hline Blue-collar worker & 0.973 & $0.435-2.175$ & 1.184 & $0.358-3.915$ & 0.881 & $0.408-1.901$ \\
\hline Low white-collar worker & 0.862 & $0.375-1.981$ & 1.187 & $0.340-4.140$ & 0.781 & $0.351-1.734$ \\
\hline Middle-high white-collar & & & & & & \\
\hline worker & 0.896 & $0.360-2.230$ & 1.008 & $0.259-3.923$ & 0.850 & $0.355-2.035$ \\
\hline Housewives/husbands ${ }^{b}$ & 1 & & & & & \\
\hline \multicolumn{7}{|l|}{ Still in work ${ }^{\mathrm{a}}$} \\
\hline Yes & 1.128 & $0.562-2.263$ & 1.533 & $0.538-4.363$ & 1.334 & $0.676-2.635$ \\
\hline $\mathrm{No}^{\mathrm{b}}$ & 1 & & & & & \\
\hline \multicolumn{7}{|l|}{ Financial support ${ }^{a}$} \\
\hline Work & 0.834 & $0.368-1.890$ & 0.720 & $0.206-2.519$ & 0.688 & $0.312-1.518$ \\
\hline Other income ${ }^{\mathrm{h}}$ & 0.751 & $0.400-1.409$ & 1.343 & $0.480-3.758$ & 0.759 & 0.413-1.392 \\
\hline Partner income & 0.841 & $0.397-1.783$ & 3.122 & $0.865-11.269$ & 0.801 & $0.387-1.655$ \\
\hline Work pension ${ }^{\mathrm{b}}$ & 1 & & & & & \\
\hline$R^{2}$ change & 10.4 & & 19.1 & & 9.7 & \\
\hline \multicolumn{7}{|l|}{ Block II (life-style) } \\
\hline \multicolumn{7}{|l|}{ Drink $^{a}$} \\
\hline Yes & 0.887 & $0.584-1.346$ & 0.865 & $0.455-1.643$ & 0.871 & $0.580-1.307$ \\
\hline $\mathrm{No}^{\mathrm{b}}$ & 1 & & & & & \\
\hline$R^{2}$ change & 0.1 & & 0.1 & & 0.1 & \\
\hline \multicolumn{7}{|l|}{ Block III (health indicators) } \\
\hline Anxiety ${ }^{a}$ & & & & & & \\
\hline Yes & $1.327^{\star}$ & $1.001-1.759$ & 1.067 & $0.706-1.613$ & $1.530^{\star}$ & $1.162-2.014$ \\
\hline $\mathrm{No}^{\mathrm{b}}$ & 1 & & & & & \\
\hline Depression $^{\mathrm{a}}$ & & & & & & \\
\hline Yes & $1.584^{\star \star}$ & $1.160-2.163$ & $1.675^{\star}$ & $1.092-2.570$ & 1.322 & $0.982-1.779$ \\
\hline $\mathrm{No}^{\mathrm{b}}$ & 1 & & & & & \\
\hline & & & & & & (continued) \\
\hline
\end{tabular}

PAGE 12 | JOURNAL OF AGGRESSION, CONFLICT AND PEACE RESEARCH | VOL. 6 NO. 12014 


\begin{tabular}{|c|c|c|c|c|c|c|}
\hline \multirow[b]{2}{*}{ Independent variables } & \multicolumn{2}{|c|}{ Minor } & \multicolumn{2}{|c|}{ Psychological abuse } & \multicolumn{2}{|c|}{ Total } \\
\hline & OR & Cl 95\% & OR & $\mathrm{Cl} 95 \%$ & OR & Cl 95\% \\
\hline \multicolumn{7}{|l|}{ Somatic symptoms ${ }^{a}$} \\
\hline Yes & 0.971 & $0.635-1.484$ & 0.664 & 0.343-1.286 & 0.983 & $0.654-1.476$ \\
\hline $\mathrm{No}$ & 1 & & & & & \\
\hline Physical diseases ${ }^{\mathrm{c}, \mathrm{i}}$ & 1.010 & $0.888-1.148$ & 1.236 & $0.995-1.534$ & 1.022 & $0.903-1.157$ \\
\hline Health care use $e^{c, j}$ & 0.969 & $0.911-1.032$ & 0.948 & $0.862-1.042$ & 0.991 & 0.933-1.053 \\
\hline$R^{2}$ change & 5 & & 4.2 & & 4.7 & \\
\hline \multicolumn{7}{|c|}{ Block IV (perpetrator characteristics) } \\
\hline \multicolumn{7}{|c|}{ Perpetrators ${ }^{a}$} \\
\hline Near one's ${ }^{k}$ & $2.355^{\star \star \star \star}$ & $1.651-3.361$ & 1.613 & $0.906-2.873$ & $2.387^{\star \star \star \star}$ & $1.696-3.361$ \\
\hline Not near ${ }^{\mathrm{b}}$ & 1 & & & & & \\
\hline \multicolumn{7}{|l|}{ Sex of perpetrators ${ }^{a}$} \\
\hline Female & 1.331 & $0.935-1.896$ & 0.827 & $0.464-1.475$ & 1.338 & $0.950-1.886$ \\
\hline Male $e^{\mathrm{b}}$ & 1 & & & & & \\
\hline$R^{2}$ change & 4.8 & & 0.1 & & 5.2 & \\
\hline \multicolumn{7}{|l|}{ Block V (social) } \\
\hline Leisure activities & 1.009 & $0.987-1.032$ & 1.001 & $0.967-1.036$ & 1.007 & 0.985-1.029 \\
\hline \multicolumn{7}{|l|}{ Social support ${ }^{a}$} \\
\hline Yes & 0.787 & $0.547-1.133$ & 1.169 & $0.663-2.061$ & 0.763 & $0.535-1.087$ \\
\hline $\mathrm{No}^{\mathrm{b}}$ & 1 & & & & & \\
\hline$R^{2}$ change & 0.3 & & 0.1 & & 0.4 & \\
\hline Total $R^{2}$ & 20.7 & & 24.5 & & 20.1 & \\
\hline
\end{tabular}

connected with an increased likelihood of exposure to minor chronicity, and the block accounted for 5 per cent of the total variance. In the block perpetrators, close relatives (e.g. spouses/cohabitants) were more likely to have perpetrated minor chronicity, and the block account for 4.8 per cent of the total variance. The variables in the social block were not independently associated with minor chronicity, and the block accounted for 0.3 per cent of the total variance. Overall, the model accounted for 20.7 per cent of the variance in minor chronicity.

Psychological, severe. In the demographic/socio-economic block, being from Germany and Greece in relation to the reference country (Sweden) were associated with a lower likelihood of exposure to high levels of severe chronicity and females more likely. The block accounted for 19.1 per cent of the total variance. The block life-style, using alcohol, was not independently associated with severe chronicity and it accounted for 0.1 per cent of the total variance. In the block health indicators, suffering from possible/probable cases of depression was connected with an increased likelihood of exposure to severe chronicity, and the block accounted for 4.2 per cent of the total variance. The variables in the blocks perpetrators and social were not independently associated with exposure to severe chronicity, and each block account for 0.1 of the total variance. Overall, the model accounted for 24.5 per cent of the variance in severe chronicity.

Psychological, total. In the demographic/socio-economic block, being from Germany, Greece, Italy, Portugal and Spain in relation to the reference country (Sweden) were associated with a lower likelihood of exposure to high levels of total chronicity and females more likely. The block accounted for 9.7 per cent of the total variance. The block life-style, using alcohol, was not independently associated with total chronicity and it accounted for 0.1 per cent of the total variance. In the block health indicators, only suffering from possible/probable cases of anxiety was connected with an increased likelihood of exposure to total chronicity, and the block accounted for 4.7 per cent of the total variance. In the block perpetrators, those close to the respondents (e.g. spouses/cohabitants) were more likely to have perpetrated total chronicity, and the block account for 5.2 per cent of the total variance. The variables in the social block were not independently associated with total chronicity and the block accounted for 0.4 per cent of the total variance. Overall, the model accounted for 20.1 per cent of the variance in total chronicity. 
Overall, minor. As shown in Table III, in the demographic/socio-economic block, being from Germany, Greece, Italy, Portugal and Spain in relation to the reference country (Sweden) and single in relation to married/cohabitants were associated with a lower likelihood of exposure to high levels of minor chronicity and females more likely. The block accounted for 11.5 per cent of the total variance. The block life-style, using alcohol, was not independently associated with minor chronicity and it accounted for 0.1 per cent of the total variance. In the block health indicators, suffering from possible/probable cases of anxiety and depression was connected with an increased likelihood of exposure to minor chronicity, and the block accounted for

Table III Multiple block-wise logistic regression analyses of the association between demographics/socio-economics, life-style, health indicators, perpetrator factors, leisure activities, social support and chronicity by severity of overall abuse (odds ratio, $\mathrm{Cl} 95$ per cent, $\left.R^{2}\right)^{\prime}$

\begin{tabular}{|c|c|c|c|c|c|c|}
\hline \multirow[b]{3}{*}{ Independent variables } & \multirow{2}{*}{\multicolumn{2}{|c|}{ Minor }} & \multirow{2}{*}{\multicolumn{2}{|c|}{$\begin{array}{c}\text { Overall abuse } \\
\text { Severe }\end{array}$}} & \multirow{2}{*}{\multicolumn{2}{|c|}{ Total }} \\
\hline & & & & & & \\
\hline & OR & Cl 95\% & OR & $\mathrm{Cl} 95 \%$ & OR & $\mathrm{Cl} 95 \%$ \\
\hline \multicolumn{7}{|c|}{ Block I (demographics-socio-economics) } \\
\hline \multicolumn{7}{|c|}{ Country ${ }^{a}$} \\
\hline Germany & $0.416^{\star \star}$ & $0.233-0.740$ & 0.552 & $0.212-1.435$ & $0.510^{*}$ & $0.291-0.894$ \\
\hline Greece & $0.251^{\star \star \star \star}$ & $0.118-0.534$ & 0.483 & $0.144-1.621$ & $0.250^{\star \star \star \star}$ & $0.119-0.523$ \\
\hline Italy & $0.438^{\star}$ & $0.218-0.882$ & 0.359 & $0.119-1.085$ & $0.396^{\star \star}$ & $0.202-0.776$ \\
\hline Lithuania & 0.787 & $0.435-1.421$ & 1.836 & $0.697-4.839$ & 1.009 & $0.562-1.811$ \\
\hline Portugal & $0.324^{\star \star \star \star}$ & $0.179-0.585$ & 0.599 & $0.265-1.353$ & $0.335^{\star \star \star \star}$ & $0.193-0.580$ \\
\hline Spain & $0.390^{*}$ & $0.182-0.838$ & 1.076 & $0.359-3.222$ & 0.594 & $0.288-1.225$ \\
\hline Sweden ${ }^{b}$ & 1 & & & & & \\
\hline \multicolumn{7}{|l|}{$\mathrm{Age}^{\mathrm{a}}$} \\
\hline 65-69 & 1.087 & $0.675-1.750$ & 0.852 & $0.399-1.821$ & 0.997 & $0.626-1.589$ \\
\hline $70-74$ & 0.853 & $0.513-1.418$ & 0.653 & $0.303-1.411$ & 0.828 & $0.507-1.352$ \\
\hline $75-79$ & 0.696 & $0.383-1.267$ & $0.372^{*}$ & $0.158-0.877$ & 0.705 & $0.398-1.248$ \\
\hline $80-84$ & 0.566 & $0.289-1.108$ & 0.460 & $0.176-1.198$ & 0.713 & $0.382-1.331$ \\
\hline $60-64^{b}$ & 1 & & & & & \\
\hline \multicolumn{7}{|l|}{ Sex ${ }^{a}$} \\
\hline Female & $1.633^{*}$ & $1.101-2.424$ & $2.043^{*}$ & $1.128-3.701$ & $1.554^{\star}$ & $1.066-2.266$ \\
\hline \multicolumn{7}{|l|}{ Marital status ${ }^{a}$} \\
\hline Single & $0.352^{\star \star}$ & $0.162-0.762$ & 0.657 & $0.206-2.096$ & 0.553 & $0.263-1.159$ \\
\hline Divorced-separated & 1.167 & $0.650-2.095$ & 1.052 & $0.472-2.341$ & 1.287 & $0.731-2.265$ \\
\hline Widow/er & 0.644 & $0.390-1.063$ & $0.461^{*}$ & $0.217-0.982$ & 0.722 & $0.449-1.162$ \\
\hline Married-cohabitant ${ }^{\mathrm{b}}$ & 1 & & & & & \\
\hline Household size $e^{c, d}$ & 1.071 & $0.878-1.307$ & 1.105 & $0.816-1.497$ & 1.110 & $0.912-1.351$ \\
\hline \multicolumn{7}{|l|}{ Education $^{\mathrm{a}}$} \\
\hline Low $^{\mathrm{e}}$ & 1.307 & $0.756-2.261$ & 0.562 & $0.248-1.273$ & 1.199 & $0.706-2.036$ \\
\hline Middle $^{f}$ & 1.190 & $0.753-1.882$ & 0.631 & $0.321-1.242$ & 1.052 & $0.674-1.641$ \\
\hline High $^{b, g}$ & 1 & & & & & \\
\hline \multicolumn{7}{|l|}{ Profession ${ }^{a}$} \\
\hline Blue-collar worker & 1.055 & $0.488-2.279$ & 0.748 & $0.254-2.201$ & 0.866 & $0.418-1.793$ \\
\hline Low white-collar worker & 0.926 & $0.420-2.041$ & 0.628 & $0.208-1.894$ & 0.753 & $0.357-1.589$ \\
\hline $\begin{array}{l}\text { Middle-high white-collar } \\
\text { worker }\end{array}$ & 0956 & $0401-2280$ & 057 & $0164-1897$ & 0790 & $0.347-1796$ \\
\hline $\begin{array}{l}\text { Worker } \\
\text { Housewives-husbands }\end{array}$ & 1 & $0.401-2 .<00$ & - & $0.104-1.001$ & 0.150 & $0.041-1.180$ \\
\hline \multicolumn{7}{|l|}{ Still in work ${ }^{a}$} \\
\hline Yes & 1.008 & $0.518-1.959$ & 1.288 & $0.510-3.250$ & 1.079 & $0.577-2.018$ \\
\hline \multirow{2}{*}{\multicolumn{7}{|c|}{ Financial support ${ }^{a}$}} \\
\hline & & & & & & \\
\hline Work & 0.984 & $0.449-2.157$ & 0.782 & $0.257-2.379$ & 0.992 & $0.471-2.089$ \\
\hline Other income ${ }^{h}$ & 0.850 & $0.464-1.557$ & 0.696 & $0.285-1.703$ & 0.765 & $0.429-1.365$ \\
\hline Partner income & 0.875 & $0.429-1.784$ & 1.048 & $0.386-2.849$ & 0.798 & $0.408-1.561$ \\
\hline Work pension ${ }^{\mathrm{b}}$ & 1 & & & & & \\
\hline \multirow[t]{2}{*}{$R^{2}$ change } & 11.5 & & 18.9 & & 11.5 & \\
\hline & & & & & & (continued) \\
\hline
\end{tabular}

PAGE 14 | JOURNAL OF AGGRESSION, CONFLICT AND PEACE RESEARCH | VOL. 6 NO. 12014 


\begin{tabular}{|c|c|c|c|c|c|c|}
\hline \multirow[b]{2}{*}{ Independent variables } & \multicolumn{2}{|c|}{ Minor } & \multicolumn{2}{|c|}{ Overall abuse } & \multicolumn{2}{|c|}{ Total } \\
\hline & OR & Cl 95\% & OR & Cl 95\% & OR & Cl 95\% \\
\hline \multicolumn{7}{|l|}{ Block II (life-style) } \\
\hline \multicolumn{7}{|l|}{ Drink $^{a}$} \\
\hline Yes & 0.888 & $0.592-1.332$ & 1.019 & $0.558-1.860$ & 0.981 & $0.666-1.444$ \\
\hline $\mathrm{No}^{\mathrm{b}}$ & 1 & & & & & \\
\hline$R^{2}$ change & 0.1 & & 0.1 & & 0.1 & \\
\hline \multicolumn{7}{|c|}{ Block III (health indicators) } \\
\hline \multicolumn{7}{|c|}{ Anxiety ${ }^{a}$} \\
\hline Yes & $1.359^{*}$ & $1.035-1.786$ & 1.236 & $0.849-1.799$ & $1.560^{\star \star \star}$ & $1.203-2.022$ \\
\hline $\mathrm{No}^{\mathrm{b}}$ & 1 & & & & & \\
\hline \multicolumn{7}{|l|}{ Depression $^{a}$} \\
\hline Yes & $1.546^{\star \star}$ & $1.140-22.096$ & $1.651^{*}$ & $1.089-2.503$ & $1.470^{\star \star}$ & $1.100-1.963$ \\
\hline $\mathrm{No}^{\mathrm{b}}$ & 1 & & & & & \\
\hline \multicolumn{7}{|l|}{ Somatic symptoms ${ }^{a}$} \\
\hline Yes & 0.897 & $0.594-1.353$ & 0.573 & $0.312-1.054$ & 0.911 & $0.617-1.346$ \\
\hline $\mathrm{No}^{\mathrm{b}}$ & 1 & & & & & \\
\hline Physical diseases ${ }^{\mathrm{c}, \mathrm{i}}$ & 0.994 & $0.880-1.123$ & $1.420^{\star \star \star}$ & $1.157-1.741$ & 1.038 & $0.922-1.169$ \\
\hline Health care use $\mathrm{c}^{\mathrm{c}, \mathrm{j}}$ & 0.992 & $0.934-1.055$ & 0.946 & $0.865-1.035$ & 1.003 & $0.947-1.063$ \\
\hline$R^{2}$ change & 4.9 & & 7.4 & & 6.2 & \\
\hline \multicolumn{7}{|c|}{ Block IV (perpetrator characteristics) } \\
\hline \multicolumn{7}{|c|}{ Perpetrators ${ }^{a}$} \\
\hline Near one's ${ }^{k}$ & $2.674^{\star \star \star \star}$ & $1.895-3.772$ & $3.122^{\star \star \star \star}$ & $1.846-5.282$ & $2.843^{\star \star \star \star}$ & 2.049-3.944 \\
\hline Not near ${ }^{\mathrm{b}}$ & 1 & & & & & \\
\hline \multicolumn{7}{|l|}{ Sex of perpetrators ${ }^{a}$} \\
\hline Female & $1.442^{*}$ & $1.026-2.026$ & 1.322 & $0.779-2.245$ & $1.520^{*}$ & $1.094-2.111$ \\
\hline Male & 1 & & & & & \\
\hline$R^{2}$ change & 6.5 & & 6.3 & & 7.6 & \\
\hline \multicolumn{7}{|l|}{ Block V (social) } \\
\hline Leisure activities' & 1.010 & $0.989-1.032$ & 0.997 & $0.966-1.030$ & 1.008 & 0.988-1.029 \\
\hline \multicolumn{7}{|l|}{ Social support ${ }^{\mathrm{a}}$} \\
\hline Yes & 0.783 & $0.550-1.032$ & 1.065 & $0.624-1.817$ & 0.735 & $0.523-1.033$ \\
\hline $\mathrm{No}$ & 1 & & & & & \\
\hline$R^{2}$ change & 3.2 & & 0.1 & & 0.4 & \\
\hline Total $R^{2}$ & 23.3 & & 32.8 & & 25.8 & \\
\hline
\end{tabular}

4.9 per cent of the total variance. In the block perpetrators, those close to the respondents (e.g. spouses/cohabitants) and females were more likely to have perpetrated minor chronicity, and the block account for 6.5 per cent of the total variance. The variables in the social block were not independently associated with minor chronicity and the block accounted 3.2 per cent of the total variance. Overall, the model accounted for 23.3 per cent of the variance in minor chronicity.

Overall, severe. In the demographic/socio-economic block, being aged 75-79 years and widow/er in relation to age 60-64 years and married/cohabitants were associated with a lower likelihood of exposure to high levels of severe chronicity and females more likely. The block accounted for 18.9 per cent of the total variance. The block life-style, using alcohol, was not independently associated with severe chronicity and it accounted for 0.1 per cent of the total variance. In the block health indicators, suffering from possible/probable cases of depression and having many diseases (e.g. cardio-vascular) were connected with an increased likelihood of exposure to severe chronicity, and the block accounted for 7.4 per cent of the total variance. In the block perpetrators, those close to the respondents (e.g. spouses/cohabitants) were more likely to have perpetrated severe chronicity, and the block account for 6.3 per cent of the total variance. The variables in the social block were not independently associated with severe chronicity and the block accounted 0.1 per cent of the total variance. Overall, the model accounted for 32.8 per cent of the variance in severe chronicity. 
Overall, total. In the demographic/socio-economic block, being from Germany, Greece, Italy and Portugal in relation to the reference country (Sweden) were associated with a lower likelihood of exposure to high levels of total chronicity and females more likely. The block accounted for 11.5 per cent of the total variance. The block life-style, using alcohol, was not independently associated with total chronicity and it accounted for 0.1 per cent of the total variance. In the block health indicators, suffering from possible/probable cases of anxiety and depression were connected with an increased likelihood of exposure to total chronicity, and the block accounted for 6.2 per cent of the total variance. In the block perpetrators, those closed to the respondents (e.g. spouses/cohabitants) and females were more likely to have perpetrated total chronicity, and the block account for 7.6 per cent of the total variance. The variables in the social block were not independently associated with total chronicity and the block accounted 0.4 per cent of the total variance. Overall, the model accounted for 25.8 per cent of the variance in total chronicity.

\section{Discussion}

\section{Chronicity and severity of abuse by country}

Overall, our results showed that Germany had the highest chronicity means in sexual abuse; Greece in psychological, injury, sexual and overall abuse; Lithuania in psychological, injury, financial and overall abuse; Portugal in physical abuse; Spain in psychological, sexual and financial abuse; and Sweden in psychological, injury, financial and overall abuse. However, when high chronicity levels were considered Greece had the greatest percentages in physical, injury and sexual abuse; Lithuania in psychological, physical, injury and financial abuse; Portugal in psychological, physical and financial abuse; and Sweden in psychological abuse. Italy had in general the lowest chronicity means in all types of abuse. Finally, considering country ranking, on the basis of the total chronicity pattern (means and percentages) by abuse types and overall abuse, revealed that Lithuania, Greece and Sweden fared worse than Germany, Portugal, Spain and Italy.

A recent report stressed the importance of assessing the specificities of elder abuse in societies, emphasizing the potential link of country inequality and occurrence of elder maltreatment (Sethi et al., 2011). Additionally, differences in the HDI between northern and southern countries remain significant, even if the situation of southern countries has improved the past years (UNDP, 2013). Data on elder abuse against women (Luoma et al., 2011), homicides against older persons (Sethi et al., 2011) and IPV (e.g. Costa et al., 2013; Archer, 2006; Straus and Mickey, 2012) indicate an excess of violence in male dominated, Southern and/or developing countries. Pertinent are the findings of Luoma et al. (2011) and Costa et al. (2013) on elevated chronicity rates in Southern countries contrasted to Northern, although the former study concerns only women and the latter IPV generally younger ages. Therefore, in the comparison between our countries we expected to find a distinct pattern between Northern European societies and South European societies in chronicity, which was not the case. The expectation was that because for instance there is greater equity (e.g. UNDP, 2013) in northern societies and that they are considered as less risky/tolerant for elder abuse and older persons may feel less discriminated (e.g. Eurobarometer 317, 2009; van den Heuvel and van Santvoort, 2011), these countries would present lower rates of high chronicity of elder abuse. In addition, despite that all these countries signed the European Convention on preventing and combating violence against women and domestic violence in order to make Europe a zero tolerance zone, the northern countries may have in general greater ability to respond to the problems of ageing and also elder abuse (e.g. Sethi et al., 2011).

In the overall pattern of chronicity, we observed that Italy, Portugal, Spain and Germany ranked the last positions. Italy tended to present the lowest means of chronicity and percentages of high chronicity levels for any type of abuse. Moreover, there were no reported cases of physical abuse with injury, and no cases of high chronicity levels (severe/total verity forms) in physical abuse and sexual abuse and financial abuse (total severity).

We found in our prevalence studies (Lindert et al., 2013; Macassa et al., 2013) relative low rates of abuse among Italian women/men ranging from 0 to 10.4 per cent, with the highest rates in psychological abuse. Although elder abuse still needs more attention in Italy, this country is considered to be very supportive towards older persons, particularly family, that besides some

PAGE 16 | JOURNAL OF AGGRESSION, CONFLICT AND PEACE RESEARCH | VOL. 6 NO. 12014 
proactive initiatives it has values such as intergenerational and family solidarity that could explain the low rates of chronicity, but this must be further elucidated. Italy has the highest old-age dependency index in the European region but only 3 per cent of people older than 65 years are cared for in nursing or residential homes, which means that most older people live in residential homes with support of families or receive care at home (Sethi et al., 2011). However, we should mention that scenery in Italy could be different in southern regions of the country (Sethi et al., 2011). On the other hand, the reporting of low/no rates of chronicity among Italian respondents could reflect their unwillingness to disclose such experiences.

Portugal had the highest chronicity means (total severity) in physical abuse, and the second greatest percentages of high chronicity levels (total severity) in physical, sexual and financial abuse and injury. A study with only women aged 60 years and over (Luoma et al., 2011) showed for instance that 39.4 per cent were exposed to any abuse during the past year, with emotional as the most commonly reported (32.9 per cent) followed by financial abuse (16.5 per cent). A recent IPV study among women/men (Costa et al., 2013) showed that Portugal tended to have the highest chronicity levels (severe acts) in most violence types than for instance Sweden and Germany. In the last years, the issue of elder abuse has received great attention, at least in terms of community awareness of the problem. Despite that Portugal ranked the last positions in the overall pattern of chronicity, elder abuse deserves great attention by policy makers. Currently, Portugal is facing a crisis which can worsen the problem of elder abuse. In the case of Spain, this country presented the second highest chronicity means (total severity) in psychological and financial abuse and the third in sexual abuse, and the third highest percentages in high chronicity levels (total severity) in sexual abuse and injury. A previous study of elder abuse with 2,401 interviews of persons over 64 years of age from the general population (Marmolejo, 2008) found abuse rates between 0.1 per cent (sexual) and psychological abuse ( 0.3 per cent), which may be underestimated due to the operational definition of abuse. However, this study revealed that 4.6 per cent of family caregivers abused the older person on some occasion in the previous year and this rose to 5.7 per cent among caregivers looking after highly disabled and dependent older people.

Based on the initial premise, we expected that Germany to be close to other northern countries (Lithuania and Sweden). Nonetheless, Germany had the highest chronicity means (total severity) in sexual abuse and the third in physical abuse, and the third greatest percentages of high chronicity levels (total severity) in psychological, sexual and overall abuse. Germany ranked fourth in relation to the overall pattern of chronicity (means and percentages). Data from Germany concerning people aged $40-85$ years show that about one-fourth of respondents older than 60 years had been verbally abused by household members within a 12-month period, whereas only 1.3 per cent of older men and 1.6 per cent of older women had been physically abused (Rabold and Gorgen, 2007). Interestingly and contrary to our findings, the Costa et al. (2013) study on IPV among women/men found low rates of chronicity (severe acts) in Germany compared to most countries involved (e.g. Greece), suggesting different chronicity patterns between for instance young and older persons.

As we expected that Germany would be close to other northern countries (Lithuania and Sweden), a following expectation was that Greece would be similar to the other southern countries in terms of pattern of abuse chronicity. However, Greece ranked the second place in the overall chronicity pattern, presenting the highest means of chronicity (total severity) in injury, the second in physical and sexual abuse and the third in overall abuse, and the greatest percentages of high chronicity levels (total severity) in physical and sexual abuse, second in injury and third in financial abuse. Greece ranked second when considering the overall pattern of chronicity (means and percentages). There is an inherent difficulty in calling attention to this topic in Greece given that deep rooted societal values concerning the protective role of family making older persons less prone to be abused (Agathonos-Georgopoulou and Browne, 1997). This view seems to be widespread among professionals, the public and the state (Daskalopoulos et al., 2006). Although abuse is often seen as a reflection of the Mediterranean temperament (OMCT, 2002), as far as we know, only three studies have addressed the occurrence of elder abuse in Greece (Daskalopoulos et al., 2006; Lindert et al., 2013; Pitsiou-Darrough and Spinellis, 1995). Among older adults aged 60 years or more from day care centres and communities in Attica $(n=757)$, it was found rates of 
psychological abuse at 34 per cent, physical 24 per cent, material 19.2 per cent and neglect 22.8 per cent, and total abuse at 16.4 per cent (Pitsiou-Darrough and Spinellis, 1995). Daskalopoulos, Kakouros, and Stathopoulou reported, in a convenience sample of persons aged between 19 and 75 years $(n=71)$, abuse/neglect rates between 1.25 and 39.7 per cent, with neglect (39.7 per cent) and psychological abuse (31.8 per cent) as the highest. More recently, findings from ABUEL (Lindert et al., 2013) revealed abuse rates in Athens between 1.1 and 13.2 per cent, the highest being financial (4 per cent) and psychological abuse (13.2 per cent). Additionally, the Costa et al. (2013) study on IPV among women/men, found that Greece had in general the highest chronicity levels (severe acts) in most violence types and compared to all countries involved (e.g. Portugal). Thus, it seems that there is a discrepancy between societal values and the reality.

Among Swedish respondents, the highest chronicity means (total severity) were observed in financial abuse, the second in overall abuse and the third in psychological abuse and injury, and the greatest percentages of high chronicity levels (total severity) in psychological/overall abuse, and the third in physical abuse. Sweden ranked third concerning the overall pattern of chronicity (means and percentages). Findings from the 1990s revealed a one-year prevalence of elder abuse of 8 per cent (Tornstam, 1989) and a study from north of Sweden with a general population sample $(n=1,009)$ reported that 16 per cent of women and 13 per cent of men had been exposed to some form of abuse after the age of 65 years (Eriksson, 2001). Elder abuse in residential settings may be relatively common, with 11 per cent of staff reporting that they knew cases of elder abuse (Saveman et al., 1999). A study about municipal care of older people revealed that 20 per cent of care receivers in dementia care and 19 per cent in general care were subjected to abuse and threats (Josefsson et al., 2007). More recently, prevalence studies with ABUEL data (Lindert et al., 2013; Macassa et al., 2013) revealed abuse rates among women/ men between 0.5 and 29.7 per cent, with the highest rates in psychological abuse. Contrary to our findings, the Costa et al. (2013) study among women/men, found lower rates in Sweden in chronicity (severe acts) compared to most countries (e.g. Greece), indicating distinct chronicity patterns between, for example, younger and older persons.

Lithuania ranked first in relation to the overall pattern of chronicity. The older persons from Lithuania had the highest chronicity means (total severity) in psychological/overall abuse, the second in injury and the third in financial abuse, and the greatest percentages of high chronicity levels (total severity) in injury and financial abuse and the second in psychological, physical and overall abuse. Elder abuse in Lithuania has not attracted great attention, although a previous study with only women aged 60 years or more has shown (Luoma et al., 2011), for instance, that 21.8 per cent were exposed to any abuse during the past year, with emotional abuse as the most commonly reported (17.8 per cent). More recently, prevalence studies based on ABUEL data (Lindert et al., 2013; Macassa et al., 2013) revealed abuse rates among women and men between 0.3 and 24.6 per cent, with the highest rates in psychological abuse (24.6 per cent).

As shown above, the frequency of abuse (chronicity) was relatively high in most countries, particularly in Lithuania, Greece and Sweden when considering the overall pattern (means and percentages). However, the regressions on chronicity by severity in psychological/overall abuse reveal a different picture concerning the relationship between country and chronicity. Our results indicate that older persons from Sweden were at higher "risk" for being exposed to high chronicity levels than the other countries, particularly in relation to minor/total psychological and overall abuse. This may not be unexpected considering the overall pattern of chronicity (means and percentages). Also, previous prevalence studies with the same population have shown that Sweden had the highest prevalence rates of psychological and physical abuse, and high in other abuse forms, e.g. injury (Lindert et al., 2013; Macassa et al., 2013). The situation for Sweden could be reflective of a greater awareness of the existence of abuse (e.g. psychological) and of a positive/cultural attitude towards reporting abuse in this country. Moreover, as reported by Erlingsson et al. (2005) elders in Sweden identified social decline and poor government as primary causes of abuse, which may be partly linked to the recent changes in long-term care in Sweden where "reductions in institutional care and cutbacks in public services have had negative repercussions for caregivers and may explain why research shows that family caregiving is expanding" (Johansson et al., 2011). But, probably families are not ready to care 
(given that in Sweden the care of elderly people has always been mainly a public responsibility) or are not sufficiently supported in the caregiving tasks, leading to possible/further/new episodes of abuse towards the elderly. In any case, further research on the relationship between country and abuse are warranted.

\section{Chronicity and severity of abuse in relation to socio-demographics}

Being female was associated with an increased likelihood of being exposed to high chronicity levels in psychological/overall abuse in all severity forms. On the other hand, women were more likely to be the perpetrators of high chronicity in minor/total overall abuse. As far as we know, these issues have not been addressed previously among older women/men aged 60-84 years from the general population. Otherwise, studies on sex differences in elder abuse are ambiguous. Some studies indicate that women are at higher "risk" for/more likely to report abuse than men (Biggs et al., 2009; Chokkanathan and Lee, 2005; Dong et al., 2007; Krienert et al., 2009; Laumann et al., , 2008; Lowenstein et al., 2009[9]; Naughton et al., 2012), and others the opposite (Biggs et al., 2009[10]; Dong and Simon, 2010; Krienert et al., 2009[11]; Lowenstein et al., 2009[12]; Naughton et al., 2012[13]) no independent relation with sex or no differences/evenness between the sexes (Acierno et al., 2010; Amstadter et al., 2011; Lachs et al., 1997; Macassa et al., 2013; Olofsson et al., 2012; Pillemer and Finkelhor, 1988; Wu et al., 2012; Yan and Tang, 2001). Furthermore, although not pertaining to elder abuse, data concerning IPV indicate either similar perpetration rates for women and men or higher perpetration rates for women, at least regarding physical abuse (e.g. Archer, 2000; Dutton, 2012; Fiebert, 2010). The Costa et al. (2013) of IPV among women/men found also in general no differences between the sexes in chronicity. As described earlier, recent prevalence studies have shown a tendency of both genders to be involved in elder abuse experiences, but our results indicate that women were in higher "risk" for chronicity of abuse. A hypothesis, at least partly, could be that women felt more comfortable to report these events than men and, in general, may have had more complaints. However, the results may be also reflective of some elements in societies that put women in greater vulnerability, which needs further elucidation concerning older women. As to the role of older women as the main perpetrators of repeated abuse, the issue needs further clarification.

Being married/cohabitant was associated with an increased "risk" for being exposed to high chronicity levels in minor psychological/overall abuse contrasted to being single and in severe overall abuse in association with being widow/er. Additionally, persons close to the respondents (e.g. spouses/partners) were more likely to be the perpetrators regarding high chronicity levels in minor/total psychological abuse and in overall abuse (all severity forms). Prevalence data on the relationship between marriage/cohabitation and elder abuse are ambiguous, with positive (e.g. Luoma et al., 2011; Lowenstein et al., 2009) and negative findings (e.g. Lindert et al., 2013; Macassa et al., 2013; Naughton et al., 2012). On the other hand, data indicate that spouses/ partners are often perpetrators (Cooney and Mortimer, 1995; Harris, 1996; Lowenstein et al., 2009). Notwithstanding, there are no previous data concerning these issues in terms of chronicity. In both cases, our results may be coherent given that most perpetrators were spouses/partners and therefore the probability of being more often involved in chronicity of psychological/overall abuse is higher among those respondents who were married/cohabitant[14]. Thus, the role of spouses/ cohabitants and other significant persons in abuse could be particularly evident regarding the frequency of abuse.

Young respondents (aged 60-64 years) were at higher "risk" for being exposed to high chronicity levels in severe overall abuse contrasted to those aged 75-79 years, which could be due to that younger respondents may have been more involved in activities and situations where repeated abuse was more likely to occur. Younger age seems to be a robust predictor of the occurrence of IPV, with younger couples experiencing higher rates of violence and older lower, and the risk of violence decreases for each year of the couple's mean age (e.g. Cunradi et al., 2002; Jewkes, 2002; Vest et al., 2002). A similar phenomenon could also pertain to the chronicity of elder abuse. However, the issue of age and elder abuse may be more complex as some findings indicate that younger older persons are more prone to abuse or that age does not play an independent role (Acierno et al., 2010; Laumann et al., 2008; Luoma et al., 2011; Macassa et al., 
2013), whereas other studies have reported that oldest old persons are more vulnerable to abuse (e.g. Naughton et al., 2012; Tatara, 1997). Thus, further research is needed to elucidate this issue, not least with regard to chronicity.

\section{Chronicity and severity of abuse in relation to mental health}

There were significant associations of anxiety and depressive symptoms with high chronicity levels. Suffering from possible/probable cases of anxiety was associated with an increased "risk" of being exposed to high chronicity in minor/total psychological abuse and overall abuse, and possible/probable cases of depression in minor/severe psychological abuse and in minor/ severe overall abuse. A hypothesis could be that the burdens of anxiety and depression on those caring for or close to the respondents led over time to repeated abuse, a finding also reported in studies regarding the occurrences of abuse (see Dong et al., 2013 for a review). However, a recent general population study did not observe an independent relationship between depression and psychological abuse (Macassa et al., 2013). The discrepancy may be related to population characteristics (e.g. general population vs selected groups). In any case, our study seems to be the first to address the relationship between mental health and chronicity of abuse, but further research is necessary to scrutinize the issue more deeply.

\section{Strengths and limitations}

To be best of our knowledge, our study is the first to address the chronicity of different abuse types in samples of older women/men from the general population in different countries and with an operational definition of chronicity of abuse that has gained wide acceptance due to its reliability/validity (Straus et al., 1996; Straus and Mickey, 2012). We have previously tested the validity and reliability of the instrument across countries and results supported the cross-country comparison (Lindert et al., 2013; Macassa et al., 2013; Melchiorre et al., 2013).

This study has several limitations that must be acknowledged. First, conclusions about causality cannot be made in view of the cross-sectional nature of the data. Thus, caution must be exercised in the interpretation of the findings. Second, the respondents were recruited in urban centres from seven specific European countries, and may not be representative for respondents from non-urban areas as well as other countries. Third, the gathered data were dependent on the respondents' subjective assessments of their situation, and were not confirmed with objective measures. For instance, the presence of probable cases of depression and anxiety was not objectively confirmed with established diagnostic instruments. Consequently, our results should be interpreted with caution. Fourth, refusal rates varied between the cities and the total refusal was high, which could have led to the "selection" of respondents diverging from people in general (e.g. more severely ill persons may have refused). However, no major differences were found between the respondents and the reference population in the community census database (age/sex). However, no major differences were found between the respondents and the reference population in the community census database (age/sex). Fifth, the variance explained by our regressions was relatively modest, indicating that other factors may also play role. Despite these limitations, the current study may have provided new insights into the experience of abuse, in particular chronicity among women/men.

\section{Conclusions}

Exposure for many acts of abuse was relatively common in several of the assessed countries, but Lithuania, Greece and Sweden fared worse as to the overall pattern of chronicity. Although respondents in most countries were exposed to many episodes of abuse, the odds of reporting above the median frequency of psychological abuse and overall abuse acts showed that respondents from Sweden tended to be more vulnerable to exposure to high chronicity levels in most of the severity forms.

Our data revealed that many older persons are exposed to abuse at frequency and severity levels, which are likely to cause great suffering. Thus, chronicity/severity of abuse and related factors calls for urgent actions in form of information campaigns, and prevention and treatment intervention from among others social/health care planers/providers. Society at society at large 
must be thoroughly informed about elder abuse. The burdens of abuse and related factors must be alleviated, protection must be given to avoid further abuse among those who have been victimized and those at risk must be identified. A successful and effective "management" of elder abuse is likely to require synchronous actions at various levels, different approaches, varying time frames and by various professionals. In this context, our results may be particularly useful for social/health care planers/providers in their work to improve the situation of abused older persons and their well-being in Europe. Furthermore, social/health care staff must be trained to probe for elder abuse, with particular attention to chronicity/severity as part of their screening of older persons that visit their services, and when abuse is present refer the older persons to proper sites.

\section{Implications for practice}

- Results provide further insights into the experience of chronicity and severity among older persons from different cultures and countries.

- Results may be helpful for advocacy and legal issues concerning elder abuse as well as to inform the public and modify their perceptions of the phenomenon.

- Results may be helpful for social/health policy makers and social/health care planers/providers in their efforts to prevent abuse and treat those who have been abused, but also to help older persons in other areas (e.g. mental health).

- Results may serve as an incitement for further investigation across cultures and countries, particularly concerning the chronicity/severity of abuse, considering factors such as mental health and the role of persons close to the victimized elders.

\section{Notes}

1. Differences do exist, for example, between countries.

2. Submitted to WHO bulletin.

3. Albeit representative and proportional to sex and age.

4. PF and PFR are used to describe and analyse heterogeneity between countries.

5. In all cases, severity pertains to the seriousness of the abuse and capacity to cause harm.

6. Total severity: both minor and severe acts together.

7. Standard glasses of wine and vodka, etc.

8. The data set was available for processing in 2011 after input, etc. Respondents who did not want a face-toface interview could self-respond and a questionnaire was sent to their homes. The self-response percentages were 38 per cent for Germany, 0.5 per cent for Greece, 0 per cent for Italy and Spain, 24.8 per cent for Lithuania, 2.3 per cent Portugal and 63.9 per cent for Sweden. Analyses (data not shown here) of response patterns contrasting interviews and self-response showed no differences (see also Fraga et al., 2013).

9. Arab women more abused than men.

10. Men more financially abused than women.

11. Men more exposed to aggravated assaults than women.

12. Jewish men more abused than women.

13. Men in the oldest age group (80 and over) more abused than women.

14. In total, 52 per cent of spouses/partners were involved in psychological abuse (high chronicity) and 50.5 per cent in overall abuse (high chronicity). In total, 67.9 per cent of near one's were spouses/partners.

\section{References}

Abath, M.B., Leal, M.C.C., Melo Filho, D.A. and Marques, A.P.O. (2010), "Physical abuse of older people reported at the Institute of Forensic Medicine in Recife, Pernambuco State, Brazil”, Cadernos Saúde Pública, Vol. 26 No. 9, pp. 1797-806. 
Acierno, R., Hernandez, M.A., Amstadter, A.B., Resnick, H.S., Steve, K., Muzzy, W. and Klipatrick, D.G. (2010), "Prevalence and correlates of emotional, physical, sexual, and financial abuse and potential neglect in the United States: the National Elder Mistreatment Study", American Journal of Public Health, Vol. 100 No. 2, pp. 292-7.

Agathonos-Georgopoulou, H. and Browne, K.D. (1997), "The prediction of child maltreatment in Greek families”, Child Abuse and Neglect, Vol. 21 No. 8, pp. 721-35.

Amstadter, A.B., Cisler, J.M., McCauley, J.L., Hernandez, M.A., Muzzy, W. and Acierno, R. (2011), Do Incident And Perpetrator Characteristics Of Elder Mistreatment Differ By Gender Of The Victim? Results from the National Elder Mistreatment Study, Department of Psychiatry, Virginia Commonwealth University, Richmond, VA.

Archer, J. (2000), "Sex differences in aggression between heterosexual partners: a meta-analytic review", Psychological Bulletin, Vol. 126 No. 5, pp. 651-80.

Archer, J. (2006), "Cross-cultural differences in physical aggression between partners: a social-role analysis", Personality and Social Psychology Review, Vol. 10 No. 2, pp. 133-53.

Babor, T.F., Higgins-Biddle, J.C., Saunders, J.B. and Monteiro, M.G. (2001), The Alcohol Use Disorders Identification Test: Guidelines for use in Primary Care, 2nd ed., World Health Organization Department of Mental Health and Substance Abuse, Geneva.

Bennett, G. and Kingston, P. (1993), Elder Abuse: Concepts, Theories and Interventions, Chapman and Hall, New York, NY.

Biggs, S., Manthorpea, J., Tinkera, A., Doyleb, M. and Erens, B. (2009), "Mistreatment of older people in the United Kingdom: findings from the first national prevalence study", Journal of Elder Abuse \& Neglect, Vol. 21 No. 1, pp. 1-14.

Boyd, C.M., Darer, J., Fried, L.P., Boult, C. and Wu, A.W. (2005), "Clinical practice guidelines and quality of care for older patients with multiple comorbid diseases: implications for pay for performance”, JAMA, Vol. 29 No. 6, pp. 716-24.

Buber, I., Kuhn, M., Philipov, D., Prskawetz, A. and Schuster, J. (2010), The Economic Situation of Older Cohorts in Europe, Vienna Institute of Demography of the Austrian Academy of Sciences, Vienna.

Centrum för folkhälsa. Stockholms läns landsting (2007), Folkhälsorapport. Folkhälsan i Stockholms län (Public Health Report, Public Health in Stockholm County), Centrum för folkhälsa, Stockholms Läns Landsting, Stockholm.

Chokkanathan, S. and Lee, A.E. (2005), "Elder mistreatment in urban India: a community based study", Journal of Elder Abuse and Neglect, Vol. 17 No. 2, pp. 45-61.

Cooney, C. and Mortimer, A. (1995), "Elder abuse and dementia: a pilot study", International Journal of Social Psychiatry, Vol. 41 No. 4, pp. 276-83.

Cooper, C., Selwood, A.C. and Livingston, G. (2008), "The prevalence of elder abuse and neglect: a systematic review", Age Ageing, Vol. 37 No. 2, pp. 151-60.

Cornwell, E. and Waite, L. (2009), "Social disconnectedness, perceived isolation, and health among older adults”, Journal of Health and Social Behavior, Vol. 50 No. 1, pp. 31-48.

Costa, D., Soares, J.F.F., Jutta Lindert, J., Hatzidimitriadou, E., Karlsson, A., Sundin, O., Toth, O., Ioannidi-Kapolo, E., Degomme, O., Cervilla, J. and Henrique Barros, H. (2013), "Intimate Partner Violence: a comparison of six European urban centers", WHO Bulletin (submitted).

Crimmins, E.M. and Beltrán-Sánchez, H. (2011), "Mortality and morbidity trends: is there compression of morbidity?", The Journals of Gerontology Series B: Psychological Sciences and Social Sciences, Vol. 66 No. 1, pp. $75-86$.

Cunradi, C.B., Caetano, R. and Schafer, J. (2002), "Socioeconomic predictors of intimate partner violence among white, black, and Hispanic couples in the United States”, Journal of Family Violence, Vol. 17 No. 4 , pp. 377-89.

Daskalopoulos, M.D., Kakouros, A. and Stathopoulou, G. (2006), "Perspectives on elder abuse in Greece", Journal of Elder Abuse and Neglect, Vol. 18 No. 2, pp. 87-104.

Dong, X., Chen, R., Chang, E.S. and Simon, M. (2013), "Elder abuse and psychological well-being: a systematic review and implications for research and policy - a mini review", Gerontology, Vol. 59 No. 2, pp. 132-42.

PAGE 22 | JOURNAL OF AGGRESSION, CONFLICT AND PEACE RESEARCH $\mid$ VOL. 6 NO. 12014 
Dong, X.Q. and Simon, M.A. (2008), "Is greater social support a protective factor against elder mistreatment?", Gerontology, Vol. 54 No. 6, pp. 381-8.

Dong, X.Q. and Simon, M.A. (2010), "Health and aging in a Chinese population: urban and rural disparities", Geriatrics and Gerontology International, Vol. 10 No. 1, pp. 85-93.

Dong, X.Q., Simon, M.A. and Gorbien, M.J. (2007), "Elder abuse and neglect in an urban Chinese population", Journal of Elder Abuse \& Neglect, Vol. 19 Nos 3-4, pp. 79-96.

Dong, X.Q., Simon, M., De Leon, C.M., Fulmer, T., Beck, T., Hebert, L., Dyer, C. Paveza, G. and Evans, D. (2009), "Elder self-neglect and abuse and mortality risk in a community-dwelling population", JAMA, Vol. 302 No. 5, pp. 517-26.

Dutton, D.G. (2012), "The case against the role of gender in intimate partner violence", Aggression and Violent Behavior: A Review Journal, Vol. 17 No. 1, pp. 99-104.

Dykstra, P.A., Van Tilburg, T.G. and De Jong Gierveld, J. (2005), "Changes in older adult loneliness: results from a seven-year longitudinal study”, Research on Aging, Vol. 27 No. 6, pp. 725-47.

Eriksson, E. (2001), Ofrid? Våld mot alder kvinnor och män- en omfångsundersökning I Umeå kommun, Crime Victim Compensation and Support Authority, Umeå.

Eurobarometer 317 (2009), Discrimination in the EU in 2009, Special Eurobarometer Report 317, European Commission, Brussels.

Erlingsson, C., Saveman, B. and Berg, A. (2005), "Perceptions of elder abuse in Sweden: voices of older persons", Brief Treatment and Crisis Intervention, Vol. 5 No. 2, pp. 213-27.

Fiebert, M.S. (2010), "References examining assaults by women on their spouses or male partners: an annotated bibliography", Sexuality \& Culture, Vol. 14 Nos 3-4, pp. 49-91.

Fraga, S., Lucas, R., Costa, D. and Barros, H. (2013), "Interviewer effects when investigating abuse were not compatible with effect modification but instead with confounding", Journal of Clinical Epidemology, Vol. 66 No. 8, pp. 911-18.

Gunzelmann, T., Schumacher, J. and Brahler, E. (1996), "Physical complaints in old age: standardization of the Giessen Complaint Questionnaire GBB-24 in over 60-year-old patients", Zeitschrift fur Gerontologie und Geriatrie, Vol. 29 No. 2, pp. 110-8.

Harris, S. (1996), "For better or worse: spouse abuse grown old", Journal of Elder Abuse \& Neglect, Vol. 8 No. 1, pp. 1-33.

IBM Corporation (1989/2011), IBM SPSS Advanced Statistics 20, IBM, Chicago, IL.

Jewkes, R. (2002), “Intimate partner violence: causes and prevention”, Lancet, Vol. 359 No. 9315, pp. 1423-9.

Johansson, L., Long, H. and Parker, M.G. (2011), "Informal caregiving for elders in Sweden: an analysis of current policy developments", Journal of Aging and Social Policy, Vol. 23 No. 4, pp. 335-53.

Josefsson, K., Sonde, L. and Wahlin, T.B. (2007), "Violence in municipal care of older people in Sweden as perceived by registered nurses", Journal of Clinical Nursing, Vol. 16 No. 5, pp. 900-10.

Krienert, J.L., Walsh, J.A. and Turner, M. (2009), "Elderly in America: a descriptive study of elder abuse examining National Incident Based Reporting System (NIBRS) data, 2000-2005", Journal of Elder Abuse and Neglect, Vol. 21 No. 4, pp. 325-45.

Lachs, M.S., Williams, C.S., O’Brien, S., Hurst, L. and Horwitz, R. (1997), "Risk factors for reported elder abuse and neglect: a nine-year observational cohort study", The Gerontologist, Vol. 37 No. 4, pp. 469-74.

Lachs, M.S., Williams, C.S., O'Brien, S., Pillemer, K.A. and Charlson, M.E. (1998), "The mortality of elder mistreatment", JAMA, Vol. 280 No. 5, pp. 428-32.

Laumann, E.O., Leitsch, S.A. and Waite, L.J. (2008), "Elder mistreatment in the United States: prevalence estimates from a nationally representative study", Journals of Gerontology Series B: Psychological Sciences and Social Sciences, Vol. 63 No. 4, pp. 248-54.

Lelkes, O. and Gasior, K. (2012), Income Poverty and Social Exclusion in the EU Situation in 2008 and Trends (based on EU-SILC 2005-2009), Policy Briefing January 2012, European Centre for Social Welfare Policy and Research, Vienna. 
Lindert, J., Luna, J., Torres-Gonzalez, F., Barros, H., Ioannidi-Kapolou, E., Quattrini, S., Stankunas, M. and Soares, J.J. (2012), "Study design, sampling and assessment methods of the European study abuse of the elderly in the European region", The European Journal of Public Health, Vol. 22 No. 5, pp. 662-6.

Lindert, J., de Luna, J., Torres-Gonzales, F., Barros, H., loannidi-Kopolou, E., Melchiorre, M.G., Macassa, G. and Soares, J.F. (2013), "Abuse and neglect of older persons in seven cities in seven countries in Europe: a cross-sectional community study", International Journal of Public Health, Vol. 58 No. 1, pp. 121-32.

Lowenstein, A., Eisikovts, Z., Band-Winterstein, T. and Enosh, G. (2009), "Is elder abuse and neglect a social phenomenon: data from the first national prevalence survey in Israel”, Journal of Elder Abuse and Neglect, Vol. 21 No. 3, pp. 253-77.

Luoma, M.-L., Koivusilta, M., Lang, G., Enzenhofer, E., De Donder, L., Verte, D., Reingarde, J., Tamutiene, I., Alves, J.F., Santos, A.J. and Penhale, B. (2011), Prevalence Study of Abuse and Violence against Older Women. Results of a Multi-cultural Survey in Austria, Belgium, Finland, Lithuania, and Portugal (European Report of the AVOW Project), National Institute for Health and Welfare (THL), Helsinki.

Macassa, G., Sundin, O., Viitasara, E., Barros, H., Torres-Gonzales, F., loannidi-Kapolou, E., Melchiorre, M.G., Lindert, J., Stankunas, M. and Soares, J.J.F. (2013), "Psychological abuse among older persons in Europe: a cross-sectional study", Journal of Aggression, Conflict and Peace Research, Vol. 5 No. 1, pp. 16-34.

Marmolejo, I.I. (2008), "Elder abuse in the family in Spain", available at: www.inpea.net/images/ Spain_Repsotr_2008_Elder.pdf (accessed 30 November 2011).

Melchiorre, M.G., Chiatti, C., Lamura, G., Torres-Gonzales, F., Stankunas, M., Lindert, J., Ioannidi-Kapolou, E., Barros, H., Macassa, G. and Soares, J.F. (2013), "Social support, socio-economic status, health and abuse among older people in seven European countries", PLoS One, Vol. 8 No. 1, p. e54856.

Naughton, C., Drennan, J., Lyons, I., Lafferty, A., Treacy, M., Phelan, A., O'Loughlin, A. and Delaney, L. (2012), "Elder abuse and neglect in Ireland: results from a national prevalence survey", Age Ageing, Vol. 41 No. 1, pp. 98-103.

O'Keeffe, M., Hills, A., Doyle, M., McCreadie, C., Scholes, S. and Constantine, R. et al. (2007), UK Study of Abuse and Neglect of Older People: Prevalence Survey Report, the National Centre for Social Research and King's College London, London.

Olofsson, N., Lindqvist, K. and Danielsson, I. (2012), "Fear of crime and psychological and physical abuse associated with ill health in a Swedish population aged 65-84 years", BMC Public Health, Vol. 126 No. 4, pp. 358-64.

OMCT (2002), Greek Helsinki Monitor and the World Organisation Against Torture. Violence against Women in Greece, World Organisation Against Torture, Geneva.

Peng, C.-H.J., Lee, K.L. and Ingersoll, G.M. (2002), "An introduction to logistic regression analysis and reporting", The Journal of Educational Research, Vol. 96 No. 1, pp. 3-14.

Pillemer, K. and Finkelhor, D. (1988), "The prevalence of elder abuse: a random sample survey", Gerontologist, Vol. 28 No. 1, pp. 51-7.

Pitsiou-Darrough, E.N. and Spinellis, C.D. (1995), "Mistreatment of the elderly in Greece", Journal of Elder Abuse and Neglect, Vol. 6 Nos 3-4, pp. 45-64.

Rabold, S. and Gorgen, T. (2007), "Abuse and neglect of older care recipients in domestic settings - a survey among nurses of in-home care services”, Zeitschrift fur Gerontologie und Geriatrie, Vol. 40 No. 5, pp. 366-74.

Saveman, B.I., Astrom, S., Bucht, G. and Norberg, A. (1999), "Elder abuse in residential settings in Sweden", Journal of Elder Abuse and Neglect, Vol. 10 Nos 1-2, pp. 43-60.

Sethi, D., Wood, S., Mitis, F., Bellis, M., Penhale, B., Marmolejo, I.I., Lowenstein, A., Manthorpe, G. and Kärki, F.U. (2011), European Report on Preventing Elder Maltreatment, World Health Organization, Copenhagen.

Straus, M.A. and Mickey, E.L. (2012) "Reliability, validity, and prevalence of partner violence measured by the conflict tactics scales in male-dominant nations”, Aggression and Violent Behavior, Vol. 17 No. 5, pp. 463-474.

Straus, M.A., Hamby, S.L., BoneyMcCoy, S. and Sugarman, D.B. (1996), "The revised conflict tactic scales (CTS2) - development and preliminary psychometric data”, Journal of Family Issues, Vol. 17 No. 3, pp. 283-316.

Tatara, T. (1997), The National Elder Abuse Incidence Study: Executive Summary, Human Services Press, New York, NY. 
Tornstam, L. (1989), "Abuse of the elderly in Denmark and Sweden: results from a population study", Journal of Elder Abuse and Neglect, Vol. 1 No. 1, pp. 35-44.

UNDP (United Nations Development Programme) (2013), Human Development Report 2013. The Rise of the South: Human Progress in a Diverse World.

van den Heuvel, W.J.A. and van Santvoort, M.M. (2011), "Experienced discrimination amongst European old citizens", European Journal of Ageing, Vol. 8 No. 4, pp. 291-99.

Vest, J.R., Catlin, T.K., Chen, J.J. and Brownson, R.C. (2002), "Multistate analysis of factors associated with intimate partner violence", American Journal of Preventive Medicine, Vol. 22 No. 3, pp. 156-64.

Yan, E.C. and Tang, C.S.K. (2001), "Prevalence and psychological impact of Chinese elder abuse", Journal of Interpersonal Violence, Vol. 16 No. 11, pp. 1158-74.

Watson, D., Pichler, F. and Wallace, C. (2010), Second European Quality of Life Survey Subjective Well-Being in Europe, European Foundation for the Improvement of Living and Working Conditions, Dublin.

WHO (2002), The world report on violence and health, World Health Organization, Geneva, Switzerland.

Wu, L., Chen, H., Hu, Y., Xiang, H., Yu, X., Zhang, T., Cao, Z. And Wang, Y. (2012), "Prevalence and associated factors of elder mistreatment in a rural community in People's Republic of China: a crosssectional study", PLoS ONE, Vol. 7 No. 3, p. e33857.

Zigmond, A.S. and Snaith, R.P. (1983), "The hospital anxiety and depression scale", Acta Psychiatrica Scandinavica, Vol. 67 No. 6, pp. 361-70.

Zimet, G.D., Powell, S.S., Farley, G.K., Werkman, S. and Berkoff, K.A. (1990), "Psychometric properties of the multidimensional scale of perceived social support", Journal of Personality Assessment, Vol. 55 Nos 3-4, pp. 610-17.

\section{Corresponding author}

Professor Joaquim J.F. Soares can be contacted at: joaquim.soares@miun.se

To purchase reprints of this article please e-mail: reprints@emeraldinsight.com Or visit our web site for further details: www.emeraldinsight.com/reprints 